\title{
SPL Color Terminology
}

National Cancer Institute

\section{Source}

National Cancer Institute. SPL Color Terminology. NCI Thesaurus. Code C54453.

Terminology used for representation of the information on pharmaceutical product color in the framework of the Structured Product Labeling documents. 